\title{
Determination of Optimal Traffic Light Period Using a Discrete Traffic Flow Model
}

\author{
Algimantas DANILEVIČIUS*, Marijonas BOGDEVIČIUS**, Modesta GUSAROVIENË***, \\ Gediminas VAIČI ŪNAS****, Robertas PEČELIŪNAS*****, Irena DANILEVIČIENË****** \\ *Vilnius Gediminas Technical University, Plytinés g. 27, 10105 Vilnius, Lithuania, \\ E-mail: algimantas.danilevicius@vgtu.lt \\ **Vilnius Gediminas Technical University, Plytinès g. 27, 10105 Vilnius, Lithuania, \\ E-mail: marijonas.bogdevicius@vgtu.lt \\ ***SI ,,Susisiekimo paslaugos”, Žolyno g. 15, 10209 Vilnius, Lithuania, E-mail: modesta.gusaroviene@vilniustransport.lt \\ ****Vilnius Gediminas Technical University, Plytinès g. 27, 10105 Vilnius, Lithuania, \\ E-mail: gediminas.vaiciunas@vgtu.lt \\ *****Vilnius Gediminas Technical University, J. Basanavičiaus g. 28, 03224 Vilnius, Lithuania, \\ E-mail: robertas.peceliunas@vgtu.lt \\ ******Vilnius Gediminas Technical University, Sauletekio al. 11, 10223 Vilnius, Lithuania, \\ E-mail: irena.danileviciene@vgtu.lt
}

crossref http://dx.doi.org/10.5755/j01.mech.24.6.22477

\section{Introduction}

During the last decades a growing number of car transport rate increasing challenge of traffic flow management. There are many different studies [1-10] that make it easier to understand traffic flow. Liu et al. [1] analyze the variation of the density of cars in a single lane using a jam control scheme based on a lattice hydrodynamic model. In this case, an additional controlled traffic flow is introduced into the traffic flow and obtained results show the changes of situation. It is controlled by only one lattice section, adjusting its traffic flow parameters. Using this method, the results are visible only after $200 \mathrm{~s}$. Also, the genetic algorithm is used for the optimization.

A modified Nagatani hydrodynamic model [2] determines the optimal traffic flow velocity estimating the traffic flow difference in information between the actual flow and the optimal flow in line [3-5]. The results were obtained by changing the response sensitivity of the traffic flow variation. The higher selected response sensitivity creates a smoother traffic flow on the road. The hydrodynamic model supplemented with delayed feedback control strategy [6-7], where according to the control traffic light the optimal vehicle velocity is determined.

Kaur et al. [8] analyzes the traffic flow in bend road with different settings using the hydrodynamic model and evaluation of driver behavior. It shows that in the case of road bends, there is a negative effect on the equilibrium of traffic flow. The aggressive driving also has a negative effect on the equilibrium of traffic flow. However, assessing the drivers' driving aggressiveness, it is possible to obtain a higher traffic flow stability by introducing bend roads. The modified Nagatani hydrodynamic model [9-10] is also supplemented by equations describing the interactions between two road lanes. Here, cars can change the lane by estimating the optimal traffic difference in lanes. In this case, the vehicle can change the lane if its lane density is higher than in the adjacent lane. As a result, the highest transport flow stability is reached when coefficient of response to the difference in density is highest. The highest concentration instability occurs when the vehicle does not have the ability to change the lane. Using the same model showed that although obtained the greatest density of stability but at the same time the velocity of the traffic flow is the lowest [11]. Hydrodynamic model investigates the aggressive maneuvering of drivers between two lanes [12]. Using the hydrodynamic model for two traffic lanes calculations were made including the probability of traffic jam formation in traffic flow [13]. The results show that although there is a high probability of traffic jam, but by combining the coefficient of the reaction to the density difference it is possible to reduce the concentration imbalance caused by the traffic jam. In [14] article hydrodynamic traffic flow model is extended with equations for optimal assessment driving uphill or downhill. It results that with the same coefficient of response to the concentration difference, but at a different angle of uphill or downhill has a very significant effect on determining the stability of the density on the road.

Jovanovic et al. [15] describes a new way to optimize traffic light parameters. It uses the Bee Colony Optimization (BCO) technique here. The aim is to reduce drivers travel time at regulated intersections.

Lee et al. [16] describes the traffic lights control method of evaluating several different traffic lanes. The problem raised in this article is a delay in traffic light control. For that purpose, the coefficients for mathematical equations that can adjust the delay characteristics in a given cycle were determined. For the detailed analysis a Kalman filter is used. The Kalman system parameters describe the relative correction factors and the lengths of the rows of stopped traffic flows beyond the turning point between $n-1$ cycle and $n+x$ cycle for future time windows.

Drivers participated in the traffic flow always want to get to the desired location as soon as possible and avoid tedious traffic jams. Therefore, in this article, using a modified discrete traffic flow model, it is aimed to determine the optimal period of traffic lights to obtain the maximum average vehicle velocity. Also to set the output boundary conditions effect for traffic flows. 


\section{Research method}

Trying to manage the urban traffic flows in the city streets it is necessary to control traffic lights period on intersections. In Vilnius one of the most crowded streets is Kalvarijų street, so it is a perfect choice for the detailed analysis and modelling the traffic flow to the city center. For the detailed analysis all simulated road is divided every 50 meters and marked with regulated intersections (Fig. 1). Calculation scheme distinguishes points that are bigger crossroads with traffic lights (indicates the (- symbol) and the points where there is no traffic light (indicates the $\mathbf{O}$ symbol).

For the analysis of traffic flow dynamics discrete traffic flow model presented in [17-18] is used. The discrete model of traffic flow uses system of equations that takes into account selected two new parameters: traffic flow density and traffic flow speed. These parameters are calculated for each road point and the information from the previous and the next point of some road mesh is taken into account (Fig. 2).

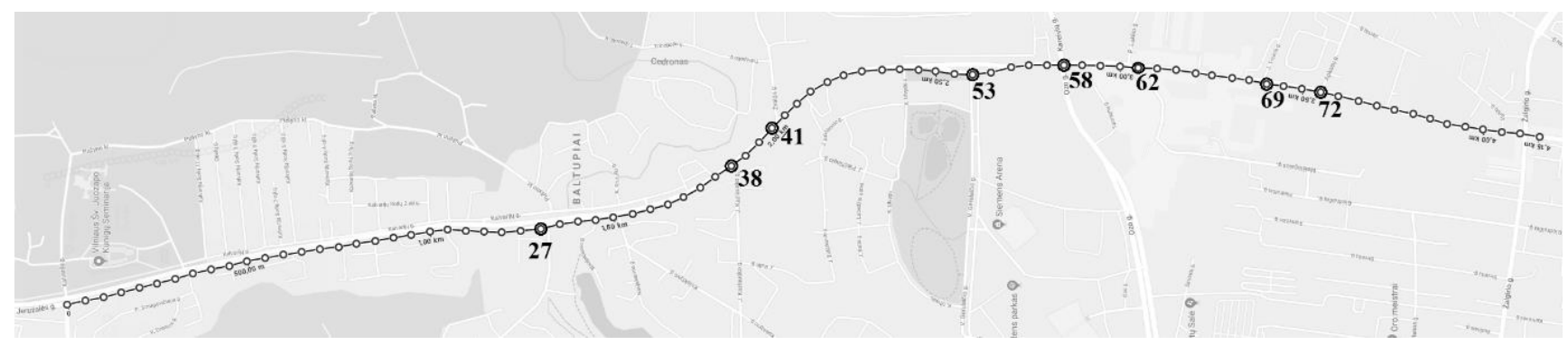

Fig. 1 One-way road section calculating scheme

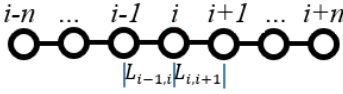

Fig. 2 Scheme to derive traffic flow values at each traffic
Eq. (1) derives the variation of traffic flow speed and equation (2) derives variation of the concentration at each point $i$ :

line point

$$
\begin{aligned}
& \dot{v}_{i}=\sum_{j=1}^{N_{\text {in }}} p_{\text {in }, i, j}(t) r_{v_{i}, i n, i, j} \Phi_{v, i n, i, j}\left(k_{j}\left(t-\tau_{i, j}\right), v_{j}\left(t-\tau_{i, j}\right), k_{i}(t), v_{i}(t)\right) v_{i}(t)- \\
& -\sum_{j=1}^{N_{\text {out }}} p_{\text {out }, i, j}(t) r_{v_{i}, \text { out }, i, j} \Phi_{v, \text { out }, i, j}\left(k_{i}(t), v_{i}(t), k_{j}(t), v_{j}(t)\right) v_{i}(t), \\
& \dot{k}_{i}=\sum_{j=1}^{N_{\text {in }}} p_{\text {in }, i, j}(t) r_{k_{i}, i n, i, j} \Phi_{k, i n, i, j}\left(k_{j}\left(t-\tau_{i, j}\right), v_{j}\left(t-\tau_{i, j}\right), k_{i}(t), v_{i}(t)\right) k_{i}(t)- \\
& -\sum_{j=1}^{N_{\text {out }}} p_{\text {out }, i, j}(t) r_{k_{i}, \text { out }, i, j} \Phi_{k, \text { out }, i, j}\left(k_{i}(t), v_{i}(t), k_{j}(t), v_{j}(t)\right) k_{i}(t),
\end{aligned}
$$

where: $k_{i}(t)$ is traffic flow density in the $i$-th point; $v_{i}(t)$ is traffic flow velocity in the $i$-th point; $p_{i n, i, j}, p_{\text {out }, i, j}$ are probabilities of flow splitting or connecting at some traffic line intersecting point $i, j ; r_{k_{i}, \text { in }, i, j}, r_{k_{i}, \text { out }, i, j}, r_{v_{i}, \text { in }, i, j}, r_{v_{i}, \text { out }, i, j}$ are coefficients of discrete model of traffic flow; $\Phi_{k, i n, i, j}\left(k_{j}\left(t-\tau_{i, j}\right), v_{j}\left(t-\tau_{i, j}\right), k_{i}(t), v_{i}(t)\right) ;$

$\Phi_{k, \text { out }, i, j}\left(k_{i}(t), v_{i}(t), k_{j}(t), v_{j}(t)\right)$;

$\Phi_{v, i n, i, j}\left(k_{j}\left(t-\tau_{i, j}\right), v_{j}\left(t-\tau_{i, j}\right), k_{i}(t), v_{i}(t)\right) ;$

$\Phi_{v, \text { out }, i, j}\left(k_{i}(t), v_{i}(t), k_{j}(t), v_{j}(t)\right)$ are nonlinear functions of discrete model of traffic flow; $N_{i n}, N_{\text {out }}$ are line numbers that incoming or outgoing the $i$-th point, respectively. Vehicle quantity at each road element is:

$$
N_{\text {auto }, e}=\int_{x_{i}}^{x_{j}} k(x) d x,
$$

where: $x_{i, j}$ is traffic line element boundary points; $k_{i, j}$ is traffic flow density at boundary points.

Coefficients $r_{k_{i}, i n, i, j}, r_{k_{i}, \text { out }, i, j}, r_{v_{i}, i n, i, j}, r_{v_{i}, \text { out }, i, j}$ of discrete model of traffic flow are equal:

$$
\begin{aligned}
& r_{k_{i}, \text { in }, i, j}=\tanh \left(c k_{\text {in }} \cdot\left(k_{i}(t) / k_{i, \max }\right)^{2}\right) ; \\
& r_{k_{i}, \text { out }, i, j}=\tanh \left(c k_{\text {out }} \cdot\left(k_{i}(t) / k_{i, \max }\right)^{2}\right) ; \\
& r_{v_{i}, \text { in }, i, j}=7.0 ; r_{v_{i}, \text { out }, i, j}=7.0 ;
\end{aligned}
$$

where $c k_{\text {in }}, c k_{\text {out }}$ are coefficients, $c k_{\text {in }}=0.15 ; c k_{\text {out }}=0.15$.

The total vehicle number in the road at time $t$ are:

$$
N_{\text {total }}(t)=\sum_{e=1}^{N_{e}} N_{\text {auto,e }},
$$

where: $N_{e}$ is number of road elements.

Starting the street's traffic simulation, input parameters are entered to the measure of the flow of transport:

$$
q_{i n}=q_{i n_{0}}+A_{q} \sin \left(\omega_{q} t\right)
$$

where: $q_{i n_{0}}$ is average value of flow, veh./s; $A_{q}$ is amplitude of flow, veh./s; $\omega$ is angular velocity, $\mathrm{rad} / \mathrm{s}$.

$$
\omega=\frac{2 \pi}{T} .
$$

$T$ is period this parameter indicates that the initial settings are entered in $T=120 \mathrm{~s}$ period.

The vehicle entered the street at the beginning of the initial speed: 


$$
v_{i n}=v_{i n_{0}}+A_{v} \sin \left(\omega_{v} t\right)
$$

where: $v_{i n_{0}}$ is average value of velocity, $\mathrm{m} / \mathrm{s} ; A_{v}$ is amplitude of velocity, $\mathrm{m} / \mathrm{s}$;

Initial values of traffic flow densities are $k_{i}=0.10$ veh. $/ \mathrm{m}$, traffic flow velocities are $v_{i}=0.10 \mathrm{~m} / \mathrm{s}$, integration time step $0.010 \mathrm{~s}$.

The following are all the traffic lights in the simulated road at the same time works in the selected periods. The calculations are carried out at different periods of traffic light entering from 98 seconds to 248 seconds. The entire period consists of half a time the red light period and half the time green light with the yellow light periods. For example 108 seconds period is determined that a yellow light lasts for 2 seconds, the green light for 50 seconds, and the red light 54 seconds (Fig. 3). As in a real system, the vehicle is moving and when the yellow light, such time selection determines that the vehicle can move and stand at traffic lights the same time.

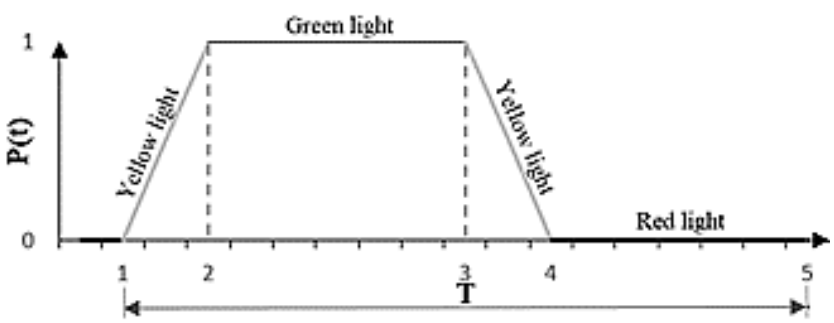

Fig. 3 The traffic light cycles

For the results, traffic flows are modeled according to the selected traffic flow density values $k_{\text {out }}=(0.05 ; 0.1 ; 0.15)$ veh. $/ \mathrm{m}$. at the last point of the simulated road. The simulation results show how the traffic flows changes in the simulated road depending on the conditions at the end of the modeled road and the frequency of traffic lights at regulated intersections.

\section{Research results and presentation of methods}

The discrete traffic flow model allows research of the dynamics of all traffic flow at any point of the simulated road by traffic lights period. The graphs of the obtained results (Fig. 4) compared the dynamics of traffic flows at the
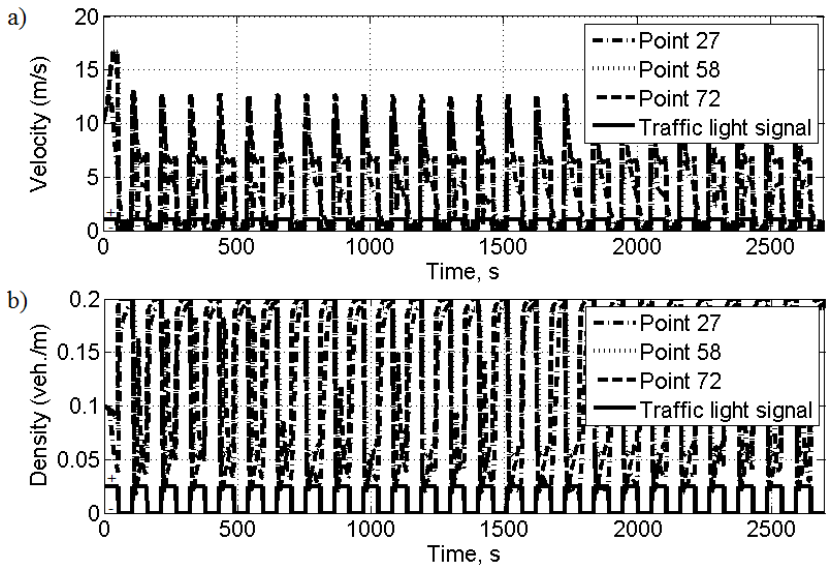

Fig. 4 The traffic flow parameters (a - velocity, b - density) variation in time at the intersections with traffic lights in $108 \mathrm{~s}$ period first regulated intersection, which corresponds to 27 th point in the model, at the highest intersection of the road which corresponds to 58th point and at the last intersection of the simulated road, which is at 72 nd point, when the traffic lights period is $T=108 \mathrm{~s}$. In graphs, traffic light status is indicated by + in green period, and - in red period.

During the first traffic light cycle, the traffic flow velocity is highest (Fig. 4, a) when the density in all points at the beginning of the simulation is 0.1 veh./m (Fig. $4, \mathrm{~b}$ ) so the modelling street is not overcrowded. After several traffic lights cycles, the traffic flow becomes steady, but over time, the density of traffic flow in the simulated street increases at 27 th point. This also affects the traffic flow velocity, which fluctuates even at a green light period. However, such fluctuations are not noticeable on simulated streets at 58 th and 72 nd points. But the density at 58 th point is higher than $72 \mathrm{nd}$ point. This suggests that small concentrations and velocity fluctuations in the simulated street at the last points cause very noticeable fluctuations in the first points, thus changing the flow parameters according to the $\mathrm{x}$-coordinate. All these changes also determine the total vehicle number in the modeled road (Fig. 5).

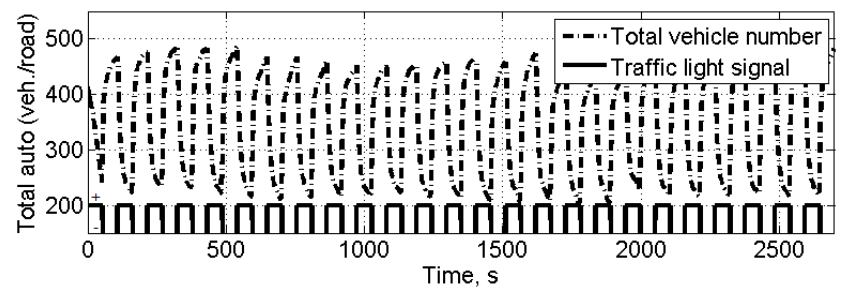

Fig. 5 The total vehicle number on the road in the traffic light 108 seconds period

The large changes in the total vehicle number in time (Fig. 5) is due to the fact that at the end of the simulated road there is no periodically stopping traffic light, so the traffic flow can move freely, and cars are introduced into the simulated road in the $T=120 \mathrm{~s}$ period. Therefore, the total vehicle number on the road constantly changes over time.

In order to determine the optimal traffic light period, the traffic flow dynamics is calculated for 11 different periods from $T=98 \mathrm{~s}$ to $T=248 \mathrm{~s}$. The average values of the traffic flow are determined, without the first $1,000 \mathrm{~s}$. Because the recent graphs show that the traffic flow in the modeled road in the first seconds of calculation is still unstable, therefore, in order to obtain the accuracy of the results, the average values are calculated in the stable mode.

After the theoretical simulations, summing up the results of velocity, flow, density and total vehicle number at different traffic light periods, it was found that the average traffic flow velocity reaches the best value at a $T=108 \mathrm{~s}$ period (Fig. 6). Reducing or increasing the traffic light period reduces the average traffic flow velocity. Although flow and density are increasing when reducing the traffic light period, but the optimal period is that, which allows traffic flow to move most faster. This increases the driver's fulfillment and reduces the negative impact on the environment and the surrounding people. As the frequent of traffic light makes drivers constantly stop and re-accelerate, which has the greatest negative impact on vehicle exhaust emissions and noise. 
Table 1

Traffic flow average values of the parameters, when $Q_{\text {in }}=0.5$ veh./s, $k_{\text {out }}=0.1$ veh. $/ \mathrm{m}$

\begin{tabular}{|c|c|c|c|c|}
\hline $\begin{array}{l}n \\
i 0 \\
0 \\
0 \\
0\end{array}$ & 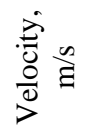 & $\frac{z}{a} \frac{\infty}{d}$ & 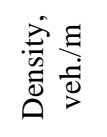 & 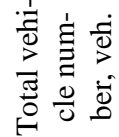 \\
\hline 98 & 3.37 & 0.1645 & 0.0856 & 354.4 \\
\hline 108 & 3.383 & 0.162 & 0.083 & 342.9 \\
\hline 128 & 3.347 & 0.161 & 0.081 & 337.2 \\
\hline 148 & 3.266 & 0.159 & 0.080 & 329.4 \\
\hline 168 & 3.280 & 0.150 & 0.073 & 303.7 \\
\hline 178 & 3.216 & 0.149 & 0.075 & 309.3 \\
\hline 184 & 3.186 & 0.147 & 0.074 & 307.5 \\
\hline 188 & 3.171 & 0.148 & 0.075 & 309.2 \\
\hline 198 & 3.202 & 0.149 & 0.074 & 306.4 \\
\hline 208 & 3.037 & 0.143 & 0.078 & 321.4 \\
\hline 248 & 3.090 & 0.145 & 0.073 & 304.9 \\
\hline
\end{tabular}
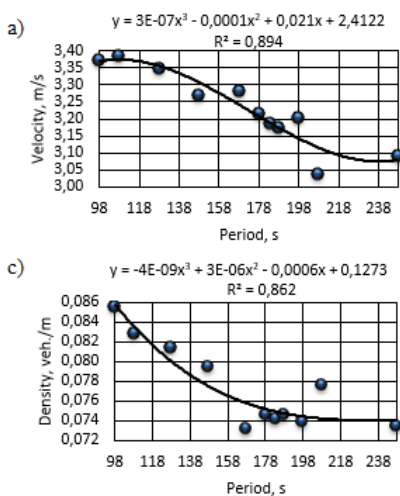

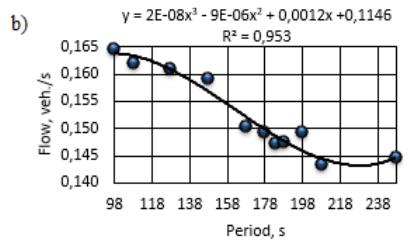

d)

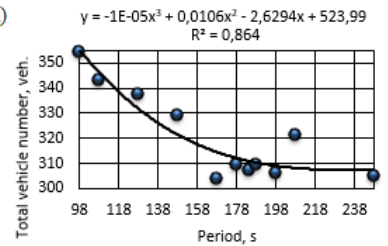

Fig. 6 The traffic flow parameters (a - velocity; b - flow; $\mathrm{c}$ - density; d - total vehicle number) dependence on traffic light period

The fundamental traffic flow curve (Fig. 7) shows the relationship between traffic flow density and flow. The obtained results show that the flow increases as density increase.

The following results are presented at a $T=108 \mathrm{~s}$ period, when it is determined that this is the optimal traffic lights period on the modeled road. Charts (Fig. 8-10) show cycles of traffic light, which include inefficient traffic flow in green traffic light period. The traffic flow starts at high acceleration in green period (Fig. 8), because after the intersection density is close to 0 veh./m. (Fig. 10).

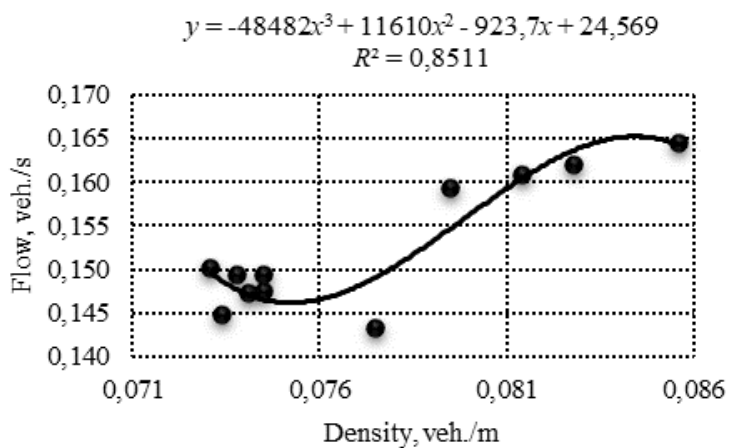

Fig. 7 Fundamental traffic flow curve
Traffic flow accelerates until the maximum permitted velocity or to the velocity of the forward moving traffic flow (Fig. 9, a). The results show that at different regulated intersections the traffic flow velocity and density differ significally (Fig. 9, b). At the last simulated intersection marked 72 nd point green light period, traffic flow reaches the set maximum speed, because there are no regulated intersections at the front. However, this velocity remains short time, due to the increasing density of traffic flow. At intersection, which is marked with 58th point, traffic flow to the maximum speed does not reach due to the traffic light in front, to which is only 200 meters away (Fig. 1). Due to the close proximity of traffic lights, the density of traffic flow at the 58th point with traffic light (Fig. 10) remains high, even in red light period. At all points from 58th to 62nd, traffic flows behave in the same way as at the intersection. But at these points, traffic flow velocity is most effective, uniformly accelerates, keeps constant velocity throughout the period of the green light and uniformly stops at a red light. This is due to the fact that the all traffic flow is moving almost simultaneously. The worst situation is at 27 th point, because the traffic flow is very non uniformly in green light period. During the period of the green light, the vehicles accelerates rapidly, due to the rapidly increasing density, the traffic flow is forced to suddenly slow down, and then decreases the density have to accelerate again. This is due to the fact that the higher traffic flow density in the front slows down the incoming flow, which can not immediately accelerate. In this way, as a wave, the increased density along the $\mathrm{x}$-axis pass through the entire traffic flow to the beginning of the simulated road, slowing the traffic flow.

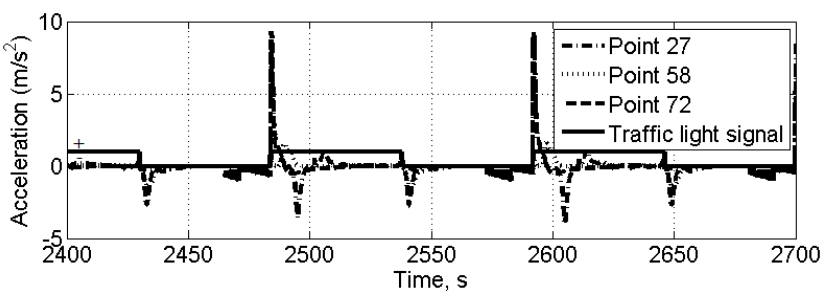

Fig. 8 Acceleration variation in time at the intersections with traffic lights in $108 \mathrm{~s}$ period
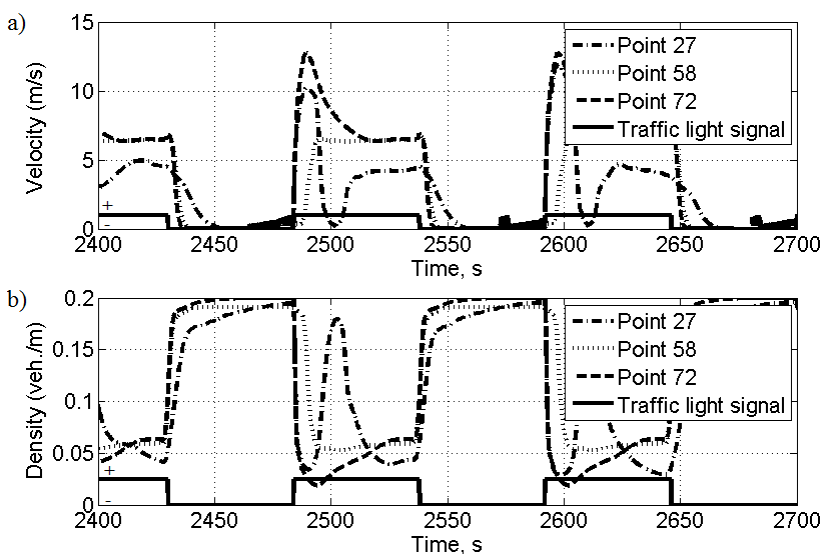

Fig. 9 The traffic flow parameters (a-velocity, b-density) variation part in time at the intersections with traffic lights in $108 \mathrm{~s}$ period 


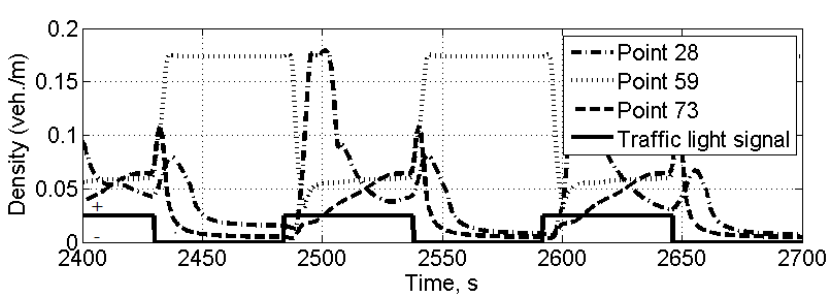

Fig. 10 Density variation part in time after the intersections with traffic lights in $108 \mathrm{~s}$ period

In order to determine the accuracy of theoretical studies corresponding to the real traffic flow, an experimental study was conducted during which the results of traffic flow velocity variation were obtained at the intersection with high traffic flow density and behind it (Fig. 11) in the green traffic light period.

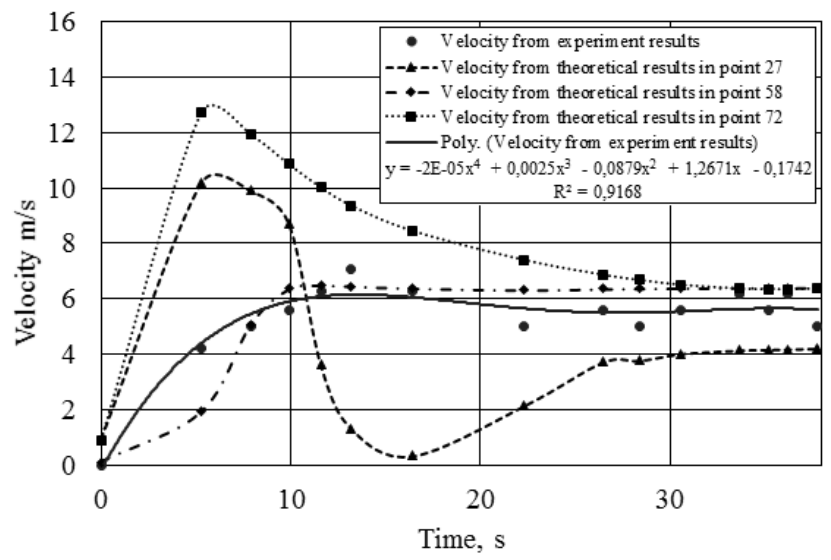

Fig. 11 Comparison of transport flow velocity variation between experimental and theoretical results in green traffic light period

Theoretical modelling results are reliable because it confirms the theoretical and experimental traffic flow velocity variation performance comparison. The average velocity variation error rate is about $6.26 \%$ comparing similar conditions in a real and simulated road. Similar conditions at the intersection marked 58th point are formed, because there is also a high density in the intersection and behind it. In other conditions, the experimental results average traffic flow velocity variation error is $20.11 \%$ compared to 27 th point and $32.86 \%$ compared to 72 nd point experimental traffic flows. However, the last two traffic flow velocity variation errors should be corrected by conducting experimental studies under appropriate conditions as in the above-mentioned points.

Fig. 12 show the results of traffic flows without first $1,000 \mathrm{~s}$ fundamental curves at different intersections of simulated road. The curves clearly show the uniformity of movement of traffic flows on a simulated road. There is a very large dispersion of results at the 27 th point intersection, which means that at the same traffic flow density, there may be very different flow. In 58th point marked intersection, there is a completely different distribution, in which there is almost no dispersal, because traffic flow here is moving uniformly.

In Fig. 13 are presented comparison of the average velocity, flow and density results of the simulated road at different traffic flow densities at the last point of the simulated road.
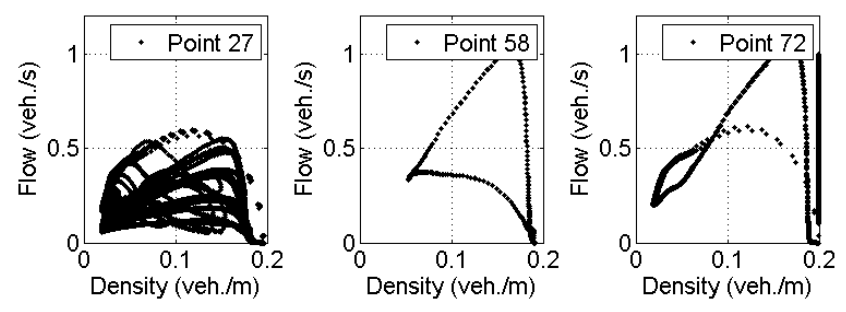

Fig. 12 Fundamental flow-density diagram in $108 \mathrm{~s}$ period

From these results it is seen that up to the density $k_{\text {out }}=0.1 \mathrm{veh} . / \mathrm{m}$ at the last point of the simulated road, does not create a traffic jam, and when the value of $k_{\text {out }}=0.1 \mathrm{veh} . / \mathrm{m}$ is exceeded, the density increases along the entire road and the velocity decreases thus affecting the resulting traffic jam on the road.
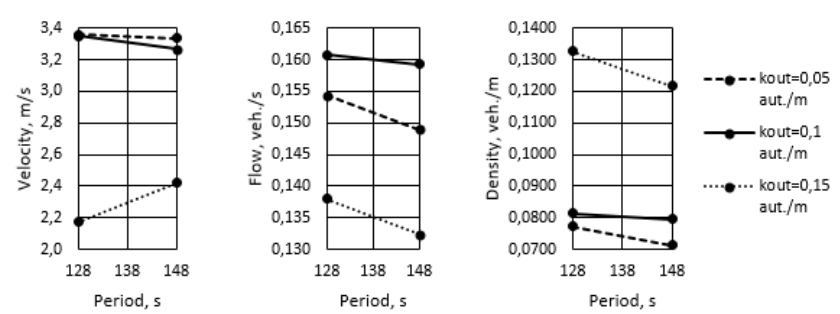

Fig. 13 Comparison of the average velocity, flow and density of the simulated road at different traffic flow densities at the last point of the simulated road

The Fig. 14 shows the total vehicle number changes on the simulated road, when the traffic lights period $T=128 \mathrm{~s}$ and the traffic flow density $k_{\text {out }}=(0.05 ; 0.1 ; 0.15)$ veh./m at the last simulated road point.

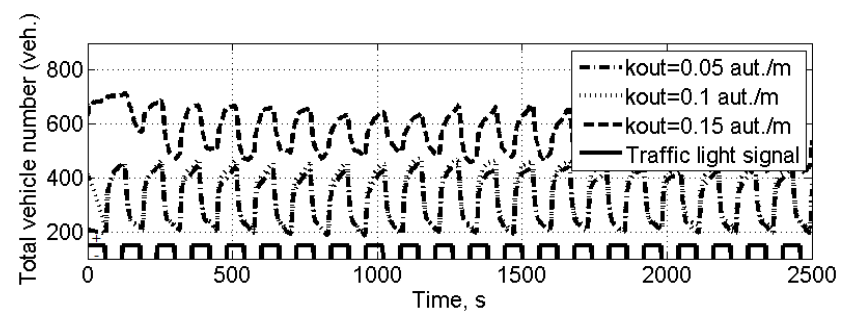

Fig. 14 Traffic flow total vehicle number variation changes chart when traffic density $k_{\text {out }}=(0.05 ; 0.1 ; 0.15)$ veh./m at simulated road last point and traffic lights period $T=128 \mathrm{~s}$

The results show that in the case of $k_{\text {out }}=0.15 \mathrm{veh} . / \mathrm{m}$, the total vehicle number on the road is twice higher than in $k_{\text {out }}=0.05 \mathrm{veh} . / \mathrm{m}$ and $k_{\text {out }}=0.1 \mathrm{veh} . / \mathrm{m}$.

The discrete model of traffic flows used to analyze the dynamical processes of rapidly changing transport flows as a solid dynamic system with changing system parameters and its own dynamic characteristics. Dynamic traffic flow characteristics can be expressed as the frequency dependence of transport flow parameters. After expanding the traffic flow parameters to time in the Furje line when the period $T=128 \mathrm{~s}$, their spectrum is obtained, which can be used to identify a particular road network natural frequencies. If traffic light control frequency is equal to the simulated traffic flow natural frequency, there is a resonance in the system. In Figs. 15-16), this is the first harmonic, which is the largest amplitude. The amplitudes of higher harmonics are smaller than the first harmonics amplitudes, which can also 
affect traffic flows. Therefore, it is important to consider which traffic control mode is better for controlling traffic flows and will the system work in resonant mode, or by contraries.

It is necessary to mention that during the same period of traffic light flow amplitudes may not necessarily decrease with each harmonic. In the second and fourth harmonics amplitudes can be higher but in the third can be very small. Velocity and density amplitude with each harmonic decreases.

After expanding the distribution of traffic flow parameters in the Furje line, according to the $T=128 \mathrm{~s}$ period, get results shows, depending on the chosen period for the control of traffic lights, frequencies change in the spectrum.
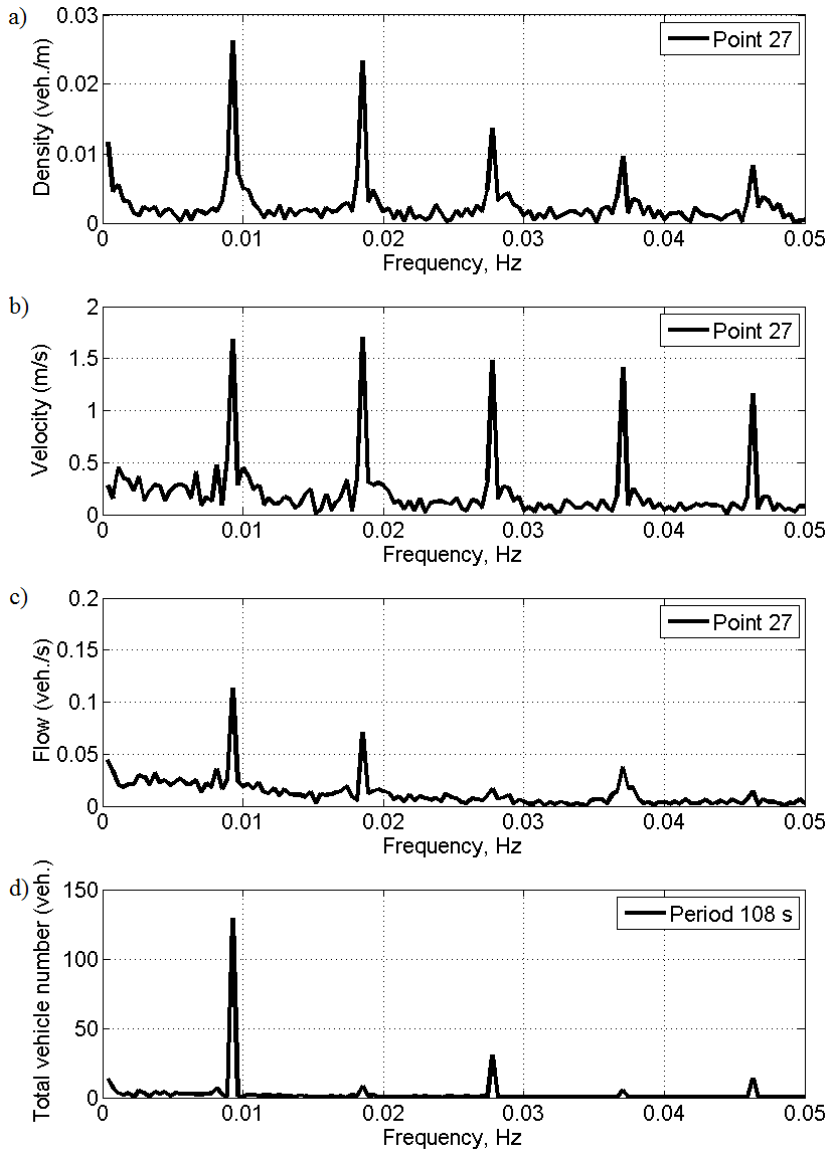

Fig. 15 The spectrum of the (a-velocity; b-flow; c-density; d-total vehicle number) amplitude at $T=128 \mathrm{~s}$ traffic lights period

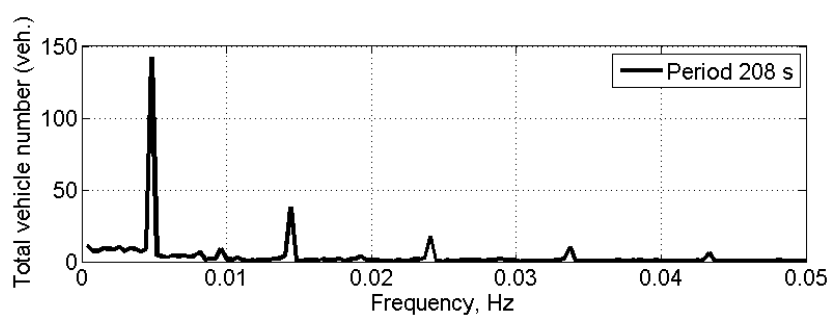

Fig. 16 The spectrum of the total vehicle number amplitude at $T=128 \mathrm{~s}$ traffic lights period

The results presented in this article are significant in that they show the possibilities of a discrete traffic flow model to precisely model transport flows under different conditions. It allows to monitor the dynamic traffic flow characteristics at any point of the simulated road and at any time. The reliability of theoretical modelling results based on experimental studies confirms the suitability of the model for using it to optimize the movement of real traffic flows. After finding the optimal traffic light period for all modeled road traffic lights operating in the same mode, further research can optimize the work of each traffic light individually due to the observed uneven traffic flow at some of the simulated road points. For this purpose, spectrum of traffic amplitude parameters could be helpful. Determining which harmonic frequency for traffic lights has a positive effect on traffic flows. Also, traffic lights period can be changed depending on the changed traffic conditions in simulated road first point and last point.

\section{Conclusions}

The modelling results allow us to analyze the dynamics of transport flows in the modeled road, depending on the studied boundary conditions, which are defined at the last point of the simulated road with traffic flow density $k_{\text {out }}=(0.05 ; 0.1 ; 0.15) \mathrm{veh} . / \mathrm{m}$ and traffic light periods from $T=98 \mathrm{~s}$ to $T=148 \mathrm{~s}$ at regulated intersections.

The results of theoretical investigations show that the optimal traffic lights period on a simulated road is $T=108$ $\mathrm{s}$, in order to get the highest average traffic flow velocity, the value of which is $3.3825 \mathrm{~m} / \mathrm{s}$.

The results of the theoretical modeling of the discrete traffic flow model have an error of about $6.26 \%$ compared to experimental results when traffic flow accelerates in green light beginning of the period.

Comparative results show that at a density of $k_{\text {out }}=$ $0.15 \mathrm{veh} . / \mathrm{m}$ at the last point of the simulated road with a case where density of $k_{\text {out }}=0.1 \mathrm{veh} . \mathrm{m}$, the total vehicle number on the road increases by $34.4 \%$, and when $k_{\text {out }}=0.05$ veh./m, the total vehicle number on the entire road decreases by $11.8 \%$

In further researches it is recommended to analyze the efficiency of the resonant and non-resonance traffic light control mode for traffic flows based on the spectrum of amplitude of traffic flow parameters.

\section{References}

1. Liu, H.; Sun, D.; Liu, W. 2016. Lattice hydrodynamic model based traffic control: a transportation cyberphysical system approach, Physica A: Statistical Mechanics and its Applications 461: 795-801. http://dx.doi.org/10.1016/j.physa.2016.06.069.

2. Nagatani, T. 1998. Modified KdV equation for jamming transition in the continuum models of traffic, Physica A 261 (3-4): 599-607. https://doi.org/10.1016/S0378-4371(98)00347-1.

3. Tian, C.; Sun, D. H.; Zhang, M. 2011. Nonlinear analysis of lattice model with consideration of optimal current difference, Commun Nonlinear Sci Numer Simulat 16: 4524.

https://doi.org/10.1016/j.cnsns.2011.03.012.

4. Gupta, A. K.; Redhu, P. 2013. Jamming transition of a two-dimensional traffic dynamics with consideration of optimal current difference, Physics Letters A 377 (3436): 2027-2033.

https://doi.org/10.1016/j.physleta.2013.06.009. 
5. Shu-Hong, Y.; Chun-Gui, L.; Xin-Lai, T.; Chuan, T. 2016. Effect of optimal estimation of flux difference information on the lattice traffic flow model, Physica A: Statistical Mechanics and its Applications 463: 394-399. https://doi.org/10.1016/j.physa.2016.07.066.

6. Wang, Y.; Cheng, R.; Ge, H. 2017. A lattice hydrodynamic model based on delayed feedback control considering the effect of flow rate difference, Physica A: Statistical Mechanics and its Applications 479: 478-484. https://doi.org/10.1016/j.physa.2017.03.027.

7. Zhu, Ch.; Zhong, Sh.; Li, G.; Ma, Sh. 2017. New control strategy for the lattice hydrodynamic model of traffic flow, Physica A: Statistical Mechanics and its Applications 468: 445-453. https://doi.org/10.1016/j.physa.2016.10.080.

8. Kaur, R.; Sharma, S. 2017. Analysis of driver's characteristics on a curved road in a lattice model, Physica A: Statistical Mechanics and its Applications. https://doi.org/10.1016/j.physa.2016.11.116.

9. Peng, G. H. 2013. A new lattice model of two-lane traffic flow with the consideration of optimal current difference, Commun Nonlinear Sci Numer Simul 18(3): 55966.

https://doi.org/10.1016/j.cnsns.2012.07.015.

10. Gupta, A. K.; Redhu, P. 2013. Analyses of driver's anticipation effect in sensing relative flux in a new lattice model for two-lane traffic system, Physica A 392: 5622. https://doi.org/10.1016/j.physa.2013.07.040.

11. Wang, T.; Gao, Z.; Zhang, W.; Zhang, J.; Li, S. 2014. Phase transitions in the two lane density difference lattice hydrodynamic model of traffic flow, Nonlinear Dyn 77 (6): 35-42. https://doi.org/10.1007/s11071-014-1325-1.

12. Li, Z.; Zhang, R.; Xu, S.; Qian, Y. 2015. Study on the effects of driver's lane-changing aggressiveness on traffic stability from an extended two-lane lattice model, Commun. Nonlinear Sci. Numer. Simul. 24 (1): 52-63. https://doi.org/10.1016/j.cnsns.2014.12.007.

13. Peng, G. H.; Liu, C. Q. 2015. Tuo M.X. Influence of the traffic interruption probability on traffic stability in lattice model for two-lane freeway, Physica A 436: 952959. https://doi.org/10.1016/j.physa.2015.05.055.

14. Jin-Liang, C.; Zhong-Ke, Sh. 2016. Analysis of a novel two-lane lattice model on a gradient road with the consideration of relative current, Communications in Nonlinear Science and Numerical Simulation 33: 1-18. https://doi.org/10.1016/j.cnsns.2015.08.025.

15. Jovanovic, A.; Nikolic, M.; Teodorovic, D. 2017. Area-wide urban traffic control: A Bee Colony Optimization approach, Transportation Research Part C 77: 329-350. https://doi.org/10.1016/j.trc.2017.02.006.
16. Lee, S.; Wong, S. C. 2017. Group-based approach to predictive delay model based on incremental queue accumulations for adaptive traffic control systems, Transportation Research Part B: Methodological 98: 1-20. https://doi.org/10.1016/j.trb.2016.12.008.

17. Bogdevičius, M.; Junevičius, R. 2014. Investigation of traffic flow dynamic processes using discrete model, Journal of KONES Powertrain and Transport 21 (4): 1519. https://doi.org/10.5604/12314005.1130421.

18. Junevičius, R.; Bogdevičius, M. 2009. Mathematical modelling of network traffic flow, Transport 24 (4): 333338.

https://doi.org/10.3846/1648-4142.2009.24.333-338.

\section{ALGIMANTAS DANILEVIČIUS, MARIJONAS BOGDEVIČIUS, MODESTA GUSAROVIENE், GEDIMINAS VAIČIŪNAS, ROBERTAS PEČELIŪNAS, IRENA DANILEVIČIENE்}

\section{DETERMINATION OF OPTIMAL TRAFFIC LIGHT PERIOD USING A DISCRETE TRAFFIC FLOW MODEL}

$\mathrm{S} \mathrm{u} \mathrm{m} \mathrm{m} \mathrm{a} \mathrm{r} \mathrm{y}$

The traffic flow problem is spread analyzed by many authors like Liu et al., Nagatani and Kaur et al. This paper investigates the traffic flow problem that occurs due to the incorrect traffic lights control. Traffic lights should be match with the traffic flow and must to optimize it. The rational traffic lights period is set by the analysis of the situation on city's street. A discrete model of traffic flow is used to obtain the results of traffic flow simulation. The results of modelling allow to analyze the dynamics of transport flows in the context of analyzed road and due to the studied boundary conditions.

In this article the discrete model of traffic flow is complemented by new parameters (coefficients), which allows to fit the modelling results with real conditions of traffic flows. Authors argued that the last point of all traffic flows is the main aspect of the investigation of the effect of the changed concentration on the modelling street. Also an amplitude spectrum of traffic flow parameters has been created. This amplitude spectrum helps to determine the frequencies of the road network.

Keywords: amplitude, density, discrete model, spectrum, traffic flow, traffic light, velocity.

Received October 15, 2018 Accepted December 12, 2018 